\title{
Sex, Intimacy and Reproductive Rights for Women with Disabilities in Zimbabwe: A Snapshot of the Views of Women with Disabilities in Harare
}

\author{
Barbra Mapuranga $^{1} \quad$ Maxwell C.C. Musingafi ${ }^{2 *}$ \\ 1.Zimbabwe Open University, Department of Special Needs Education, Faculty of Applied Social Sciences \\ 2.Zimbabwe Open University, Department of Development Studies, Faculty of Applied Social Sciences
}

\begin{abstract}
This paper is derived from study on sexual and reproductive rights of women with disabilities in Zimbabwe. The paper explores the sexual and reproductive rights (SRR) and practices vis a vis women with disabilities in Zimbabwe. This is done with the view of drawing insights and policy recommendations on sexual and reproductive rights for women with disabilities in Zimbabwe. The study argues that there are serious gaps in SRR policies and their implementation for WWDs. While the availability of acceptable and supportive public policy and legislation indicates the beginning of a commendable process of services delivery to all stakeholders, this alone is not sufficient unless supported by effective systems and processes for effective delivery. Formulation and adoption of a policy or piece of legislation does not automatically translate to its implementation and/or enforcement. The study recommends change of mind-set for both the cultural environment and law with regards what is acceptable and legal sex; domestication and enforcement of international and regional disability laws; redesign of national laws to clearly address SRRs for WWDs; communication and advocacy of disability laws to the people. follow-ups and performance evaluation of families and communities on how they treat WWDs vis a vis their SRR; and involvement of WWDs in policy formulation.
\end{abstract}

Keywords: Sex, women, disability, rights, reproductive, disability.

DOI: $10.7176 / \mathrm{JCSD} / 51-03$

Publication date:September $30^{\text {th }} 2019$

\section{Orientation}

According to Frohmader (2013), 15\% of the world's population lives with some form of disability and frequently encounter a myriad of physical and social obstacles. These figures are rapidly increasing, as a result of population growth, ageing, violence, war, conflicts, environmental degradation, poor workforce conditions, sexual and gender-based violence, harmful traditional practices, and improvements in measurement techniques (Frohmader, 2013). The most affected are women and girls. They face barriers to full equality and advancement because of such factors as race, age, language, ethnicity, culture, religion or disability (Beijing Platform for Action, 1995). Persistence of certain cultural, legal and institutional barriers makes women and girls with disabilities the victims of two-fold discrimination: as women and as persons with disabilities (Maruzani, 2016). In most cases, women with disabilities are invisible both among those promoting the rights of persons with disabilities, and those promoting gender equality and the advancement of women (Choruma, 2007).

According to Maruzani and Mapuranga (2016), disability is a complex phenomenon covering a number of diverse conditions which include activity impairment, limitation and participation restrictions. From the diverse occurrence of disability observations are that there is no universally accepted definition for disability (Palmer and Harley, 2012). Focusing its definition on comparative ways of doing things, the Disability World (2012) gives disability as a condition or function, judged to be significantly impaired relative to the usual standard of an individual or group. Arguing from a capabilities approach perspective Mitra, Posarac and Vick (2013) analyses disability as a derivation of capabilities or as a deprivation of functions. Her definition focuses more on impairment which she defines as physiological, mental or anatomical loss. It is from these impairments that deprivation manifests as the individual interacts with the resources available and the environment (Mitra, Posarac and Vick, 2013). If all these definitions are brought together disability can be given as a phenomenon which develops from bodily impairment(s) which if not properly provided for can lead to limitations in an individual's social interactions. Disability becomes more conspicuous when comparisons are made with nonimpaired individuals.

The medical model of disability defines disability as a condition caused by physical impairments resulting from disease, injury or health condition which leads to the loss of bodily and social functioning (Palmer and Harley, 2012). To help people with disabilities, in this case women, interventions are primarily linked to medical treatment and rehabilitation (Palmer and Harley, 2012).

The social model of disability defines disability as a social construct (Mitra, 2006). The model focuses on how society puts in place services to accommodate people with disabilities so that they can function without 
hassles in the society. Oliver (1996) argues that it is society which disables physically impaired people. He further adds that disability is an imposition on top of people's impairments and it manifests in the way people are unnecessarily isolated and excluded from full participation in society (Oliver, 1996). Adopting a Marxist view in explaining disability Mitra (2006) argues that people with disabilities are discriminated and segregated through sensory, attitudinal cognitive, physical and economic barriers. Such experiences are perceived as similar to those of an oppressed minority group. In the same line of thinking the British Council of Disabled People argues that disability is the disadvantage or restriction of activity caused by society which leads to their exclusion from mainstream activity (Morris, 1998). Thus, like racism or sexism disability is discrimination and social oppression.

The social model of disability de-musicalizes and de-individualises disability. Arguing against this model, Palmer and Harley (2012), criticize the social model of disability for its failure to address issues of impairment which is given as a contributing factor to the limited development of disability specific policy and secondly the limited robust research into living standards of people with disabilities. They posit that by concentrating on societal factors this model of disability contributes to the little attention being given to the role of impairment in people with disabilities' experiences.

The criticism given above led to the development of the social relational model of disability, which sees disability as a result of societal attitudes added to the effect of impairment (Palmer and Harley, 2012). This model places importance on reducing societal barriers for people with impairments.

The most current model is the international classification of functioning disability and health (ICF) which was developed by World Health Organisation (WHO) in the 1980s as an integration of the medical and social models of disability (Mitra, 2006). This was done in an attempt to come up with a coherent view of disability (WHO, 2001). The ICF model articulates disability as a health condition that leads to various impairments. The impairments then lead to activity limitations and participation restrictions in certain social contexts (Mitra, 2006).

The ICF refers to individual functioning including physical impairment, sensory impairment, cognitive and intellectual impairment and various types of chronic disease (Disability World 2015). It identifies three dimensions of disability that is, body, structure and function which refers to activity and participation. It also considers physical and social/environmental factors in affecting disability outcomes (Disability World, 2015).

Also, issues of sexuality affect women with disabilities most. Sexuality has been simply defined as sexual orientation (Peta, 2015). The most common, legal and acceptable sexual orientation among most cultures is heterosexuality (Epprecht, 1998; Zinanga, 1996). Heterosexuality is a relationship where male and female individuals have conventional sex for either procreation or pleasure (Zinanga, 1996). All other sexual orientations are neither legally nor morally acceptable in most communities (Epprecht, 2013). But, this does not mean these orientations (homosexuality, lesbians, masturbation, and etcetera) do not exist in those communities (Epprecht $(1998,2013)$. These other forms are just for pleasure not procreation, hence culturally and morally unacceptable. It thus follows that the acquisition of a husband and having children within the parameters of a heterosexual marriage awards women respect within families and communities (Zinanga, 1996). If sex is about procreation and women with disabilities are assumed incapable of making children, it follows that they are denied the pleasures associated with the sex and reproductive processes.

If there are several sexual orientations as determined by biological, cultural, ethical, legal, historical, religious, spiritual, among other factors, then it follows that sexuality is not simply about vaginal nor anal penetration by a penis. Disabled women who may not be penetrable due to the nature of their disability can still enjoy sex through appropriate forms of sexuality. This, however, may not be possible in countries like Zimbabwe since the only acceptable sexual activity is where men and women have conventional sex. As a result, women with disabilities may among other things constantly worry about whether they would be worthy lovers or whether they will be capable of having an orgasm (Barrett, 1999). Such worries erode the sexual confidence of women with disabilities in a context where they feel they will be regarded by society as "sick" people (Basson, 1998). Such women lose their self-esteem and confidence to the extent that they give up on having intimate partner relationships before they even try (Peta, 2015). Women with disabilities are thus denied their right to participate in sexual activities.

\section{Study methodology}

Qualitative research methodology forms the basis of this study. Qualitative methodology has been chosen for this study because it seeks answers from the experiences of groups or individuals. The study focused on women with disabilities in Harare and conducted in their own local settings. Qualitative methodology allowed the researchers to share in the understandings and perceptions of others and to explain how people structure and give meaning to their daily experiences. Data for this study was drawn from a combination of documented literature, oral tradition, case studies, ethnographic and phenomenological strategies based on participatory observation, key-informant and in-depth interviews, secondary review of policies and legislation, and archival records and visual data. 


\section{A global overview of women with disabilities}

It is worth noting that there are significant differences in the prevalence of disability between men and women in both developing and more developed countries. Frohmader (2013) observes that the male disability prevalence rate is $12 \%$ while the female disability prevalence rate is $19.2 \%$. However, a detailed global picture on how gender and disability intersect is not yet possible as data collection and research has been extremely limited and often clouded by factors that are quantification challenges, such as the feminisation of poverty, cultural concepts of gender roles and sexual and reproductive rights, violence, abuse and other types of exploitation, such as child labour (Frohmader and Meekosha, 2012).

It has been established that women with disabilities and men with disabilities have different life experiences due to biological, psychological, economic, social, political and cultural attributes associated with being female and male (Maruzani, 2016; Moyo, 2010). Patterns of disadvantage are often associated with the differences in the social position of women and men. These gendered differences are reflected in the life experiences of women with disabilities and men with disabilities, particularly in relation to sexual and reproductive rights and genderbased violence (Maruzani, 2016; Manjoo, 2015). Although men and women with disabilities are subjected to discrimination because of their disabilities, women with disabilities tend to suffer double discrimination due to gender and disability (Abu Habbib, 1997). While it is easy for boys / men to be tolerated and get married, the situation is different for girls /women with disabilities who are seen as a drain on already stretched resources (Maruzani, 2016).

According to Manjoo (2012), no group has ever been as restricted in respect of their reproductive rights as women with disabilities. Since women with disabilities are seen as dependent and in need of being taken care of, it is difficult for many people to imagine how a mother with a disability can fill the caring and nurturing mothering role (Shaul, Dowling and Laden, 1985). The belief that women with disabilities cannot and should not bear and raise children has made it difficult for women with disabilities to find men who will accept them. Even when women with disabilities become mothers, they encounter many difficulties because the non-disabled world assumes that disability makes them unfit to be mothers. Many lose custody of their children in divorce while others may have their children removed from their care by social welfare agencies, solely on grounds of disability (Choruma, 2007). If they manage to keep their children they often encounter difficulties in dealing with early childhood programmes that traditionally have neglected and ignored the needs of mothers with disabilities (Corbett, 1989; Maruzani, 2016).

Although society's fears that women with disabilities will produce defective children are for the most part groundless, these fears have resulted in severe discrimination against women with disabilities (Maruzani, 2016). Around the turn of the century what was referred to as "feeble-mindedness" was considered a major threat to society. "No feeble-minded mother will ever have a child absolutely normal in every respect" (Johnson, as quoted in Wolfensberger, 1975, p 38). More important, these women were thought to lack moral restraints concerning sexual activities and it was believed that as a consequence they would produce a large number of illegitimate and deficient children (Simmons, 1982). The preventative measures taken against this threat included large scale forced sterilization (Scheerenberger, 1987) and institutions for "feeble minded women of childbearing age" were established where women with mental retardation were segregated from society as well as from men with mental retardation.

It is widely documented that women with disabilities are typically seen as asexual (Asch and Fine, 1988; Finger, 1985; Matthews, 1983; Shaul, Dowling and Laden, 1985; Waxman, 1989; Moyo, 2010; Maruzani, 2016). Because women with disabilities are seen as asexual they are not seen as in need of information about birth control or what is possible in terms of having a sex life and children. Some women with disabilities have criticized the disability rights movement for not addressing sexuality as a political issue similar to housing and transportation. For example, Waxman (1989, p 2), claims that many people with disabilities consider sexuality to be the area of greatest oppression: "We are more concerned with being loved and finding sexual fulfilment than getting on a bus." Shaul, Dowling and Laden (1985) observe that people without disabilities tend to view sex as an acrobatic activity which makes it difficult for them to imagine how people with physical impairments can be sexually active.

The CRPD Committee has clearly identified that discrimination against women and girls with disabilities in areas of sexual and reproductive rights, including gender-based violence, is in clear violation of multiple provisions of the CRPD. It has explicitly articulated the urgent need for states parties to address these multiple violations. Yet such violations are still prevalent as evident in the following examples taken from Frohmader (2013): forced and/or coerced sterilisation; forced contraception; gender based violence; lack of access to sexual and reproductive health services and programmes; lack of access to information and education on sexual and reproductive rights; and lack of access to justice.

It is therefore concluded that the realisation of the rights of women with disabilities are still a long way because they have suffered and endured marginalisation since time immemorial. Even though disability movement has had a good fight in calling for the recognition of people with disabilities in various ways and for a 
number of rights, the rights of women with disabilities have not been seen to be an issue in most countries. Women with disabilities are still treated as asexual human beings who should not enjoy their sexuality and reproductive rights (Maruzani, 2016).

The CRPD recognises gender as one of the most important categories of social organisation, emphasising the need to incorporate a gender perspective in all efforts to promote the full enjoyment of human rights and fundamental freedoms by people with disabilities. It recognises that women and girls with disabilities are subject to multiple discriminations, and that states parties to the Convention have an obligation to take measures to ensure women and girls with disabilities experience the full and equal enjoyment of all human rights and fundamental freedoms. Yet despite this, people with disabilities are often treated as asexual and genderless human beings (Frohmader, 2013). This view is borne out in disability and development policies and programmes world over, which consistently fail to apply a gender and/or disability lens. Most proceed as though there are a common set of issues - and that men and women experience disability in the same way (Ibid).

Thus, by virtue of being women and disabled, women with disabilities face a double discrimination (United Nations, 2006). Deprivation of basic rights for such a group is very rampant. Their lack of access to empowerment and means of production also heavily limits their negotiating capacities and leaves them vulnerable to exploitation and abuse. Abuse also occurs because of serious lack of information to persons with disabilities (Maruzani, 2016). This is so because information is mainly presented in inaccessible formats such as Braille for the visually impaired and sign language for the hearing impaired (ibid). This coupled with societies such as the Zimbabwean where issues of sexual and reproductive rights do not form part of ordinary everyday discourse, leaves women with disabilities without any source of information (ibid).

Closely related to this is the issue of dangerous cultural practices which makes the situation of women with disabilities quite vulnerable. In some cases, persons with disabilities are viewed as asexual and therefore deprived of their right related to sexual and reproductive issues (Moyo, 2010). There are two obvious results of this thinking. On one hand, some family members of persons with disabilities may tend to become overprotective while on the other hand, some may actually neglect the family member with disabilities living them uninformed at all about their sexual and reproductive rights (Choruma, 2007). The situation gets more compounded by beliefs that sleeping with people with disabilities especially women with disabilities can be a cure for HIV or a charm for good luck in business (Flood, 2010). This means that when looking at women with disabilities and their sexual and reproductive rights, not only is one dealing with a group that is deprived of the enjoyment of these rights but a group which is also exposed to very risky situations (Moyo, 2010).

Among other things, the CRPD mandates states to protect persons with disabilities from violence, exploitation and abuse; ensure that persons with disabilities enjoy legal capacity on an equal basis with others; enjoy access to justice; are not subjected to arbitrary or unlawful interference with their privacy and family; guarantee persons with disabilities; take measures to ensure women and girls enjoy the full and equal enjoyment of their human rights; prevent people with disabilities from being subjected to torture, or cruel, inhuman or degrading treatment or punishment; prohibit involuntary treatment and involuntary confinement; and implement disability inclusive development practices and ensure the right of people with disabilities to the highest attainable standard of health without discrimination.

\section{Findings of the study}

The findings of the study revealed that like all other human and fundamental rights SRR form a core part of WWDs. Neglecting these equally important rights would be detrimental to the wellbeing of the larger part of the population of women in the world.

This study established that although the country has attempted to align its policy and legal framework with international standards, it was still lagging behind the international standards. It also established that it was not very different from the majority of other countries, especially the developing countries. The study established that there were serious gaps in terms of policy implementation. In fact, policy and practice were found to be at loggerheads. It was established that even the laws that are there in Zimbabwe are not good enough to ensure full access to SRR for WWDs as they were found not to be in conformity with the international and regional standards. The UN Convention on the Rights of Persons with Disabilities (CRPD) and the African Disability Protocol (ADP) had not been domesticated in Zimbabwe. Hence, the policy and legal framework fell far short of the international standard benchmarks.

This study revealed that WWDs experienced limited access and enjoyment of SRR because their parents and society as a whole are not aware of how to help them to enjoy these as stipulated in the international legal frameworks. The parental and societal roles have proved to be limiting and depriving as indicated by forced sterilization, contraceptives, forced marriages, rejection and divorce stereotypes as well as gender based violence. Setbacks to enjoyment of SRR by WWDs in Zimbabwe included lack of awareness by their social network which includes parents, family members and society at large. These have proved in the study to have negative attitudes towards WWDs, lack of disability etiquette and disability language. It therefore came out in this study 
that deprivation of SRR of WWDs in Zimbabwe by their parents and society is compounded by the unfocused or rather lack of disability policy.

The study established that sex is not the same as sexual intercourse. Although some of the participants said no sex before marriage, what they were referring to was conventional sexual intercourse. They were actually indulging in sex and satisfying their sexual desires through hugging, kissing and touching their male partners. The study also established that the only culturally and legally accepted sexual activity in Zimbabwe was the conventional coitus where a penis enters a vagina especially between married couples. All other forms of sexual gratification were not culturally acceptable and had been criminalised. This had ensured that some categories of WWDs (like the physically impaired) would not indulge in legal and cultural accepted sexual activities in Zimbabwe.

All female participants had experienced intimacy at some point in their lives although the forms of experiences varied as determined by the type of disability and attitudes. Not everyone had equal freedom and access to sex and reproduction. Some are just given to old men, whilst others are denied access. In fact, WWDs are not given the opportunity to decide on their sexuality, when to have sex and with whom they want to spend their sex life. In most cases, they are forced to live in celibacy without asking for their opinion.

The study established that the most common, legal and acceptable sexual orientation among Zimbabwean cultures is heterosexuality because it leads to procreation. The cultures do not accept incest, masturbation, oral sex, anal sex and homosexuality. This means that all those who are incapable of heterosexual relationships are excluded from sexual activities. Thus, if sex is about procreation and women with disabilities are assumed incapable of making children, it follows that they are denied the pleasures associated with the sex and reproductive processes. In fact, rejection of all other forms of sexual orientation other than heterosexuality means that disabled women who may not be penetrable due to the nature of their disability cannot enjoy sex in their lifetime.

The study also established that most WWDs go through forced sterilisation; forced contraception; gender based violence; lack of access to sexual and reproductive health services and programmes; lack of access to information and education on sexual and reproductive rights; and lack of justice.

Overall, WWDs are seen as asexual and therefore they do not have sexual and reproductive rights. They are forced to live without sex; go through forced sterilisation; forced contraception; gender based violence; lack of access to sexual and reproductive health services and programmes; lack of access to information and education on sexual and reproductive rights; and lack of justice. It is thus, concluded that the realisation of the rights of women with disabilities is still a long way because they have suffered and endured marginalisation since time immemorial.

\section{Recommendations}

Based on the findings discussed above, this study provides a wide range of recommendations:

- Sex and sexuality: There is need for change of mind-set for both the cultural environment and law with regards what is acceptable and legal sex. Otherwise, some WWDs remain condemned to a sexless life throughout their life time. In fact, the sex and sexuality regime needs to be liberalised so that it accepts masturbation, lesbianism, homosexuality, oral sex, among others as ways of meeting sexual needs for people who cannot access conventional sex of penis penetrating vagina so that WWDs are not segregated from indulging in sex. What this would mean is that there is need for re-orienting the mind through relevant legislation and workshops that avail information to the generality of the population.

- Disability laws: This study established that in some cases there is lack of enforcement of international and regional disability laws. It is thus, recommended that international and regional disability laws must be enforced. There is also need for domestication of these laws. The government should also ensure that the subjects of gender and sexuality are visible in local legislations. Gender blind and gender-neutral laws are not equally applicable to all WWDs.

- Advocacy and training: There is urgent need for communicating disability laws to the people. In this study, participants talked of cases of sterilisation, forced contraception, forced marriages for the WWDs. These are illegal practices which may be happening because of lack of knowledge. People working or living with WWDs also need orientation in the laws governing disability so that they understand and actively participate in the implementation of the laws and the policy framework.

- Regular reviews and check-ups: To measure progress and success in the implementation of disability laws, the Ministry and departments concerned should make follow-ups and performance evaluation of families and communities on how they treat WWDs vis a vis their SRR.

- Involvement of WWDs in policy formulation: This study recommends full engagement of WWDs in the formulation of such policy frameworks through consultation and workshops that tap their views and fears. This will resonates well with their adage "Nothing for us without us" 


\section{References}

Abu-Habib, L. (1997). Gender and disability: Women's experiences in the Middle East. Oxford, Oxfam UK and Ireland.

Asch, A., and Fine, M. (1988). Introduction: Beyond pedestals. In M. Fine \& A. Asch (Eds.), Women with disabilities: Essays in psychology, culture, and politics. Philadelphia, PA: Temple University Press.

Barrett, M. (1999). Sexuality and multiple sclerosis. Toronto: Multiple Sclerosis Society of Canada.

Basson, R. (1998). Sexual health of women with disabilities. CMAJ: Canadian Medical Association Journal, 15(4). Pp. 359.

Beijing Platform for Action (1995). Action for Equality, Development and Peace. Chapter IV, Strategic objectives and actions, paragraph 46. Fourth World Conference on Women. Beijing. Ploatform for Action.

Choruma, T. (2007), The forgotten tribe, people with disabilities in Zimbabwe, London, Progressio.

Corbett, K. (1989). Disabled mom loses kids. New Directions for Women, September/October, 1989, 4-5.

Disability World (2015) Disability Definition Types and Models .[Online ]Available from: http://www.disabled-world.com/disability/types/. [Accessed: 13 December 2015.

Epprecht, M. (1998). The 'unsaying' of indigenous homosexualities in Zimbabwe: mapping a blindspot in an African masculinity. Journal of Southern African Studies, 24(4). Pp. 631-651.

Epprecht, M. (2013). Hungochani: The history of a dissident sexuality in southern Africa (2nd ed.) Montreal, Quebec: McGill-Queen's Press-MQUP.

Finger, A. (1985). Claiming all of our bodies: Reproductive rights and disability. In S. E. Brown, D. Connors, \& N. Stern (Eds.), With the power of each breath: A disabled women's anthology. Pittsburgh, PA: Cleis Press.

Frohmader, C. (2013) 'Dehumanised: The Forced Sterilisation of Women and Girls with Disabilities in Australia'. Women with Disabilities Australia. Rosny Park, Australia.

Frohmader, C. and Meekosha, H. (2012) 'Recognition, respect and rights: Women with disabilities in a globalised world.' In Disability and Social Theory, Edited by Dan Goodley, Bill Hughes and Lennard Davis, London: Palgrave Macmillan.

Government of Zimbabwe. (2013). Constitution of Zimbabwe: Amendment (No. 20) Act (2013). Harare: Fidelity Printers and Refiners.

Government of Zimbabwe. (1992). Disabled Persons Act, Chapter 17:01. Harare: Government Printers

Manjoo, R. (2012) Report of the Special Rapporteur on violence against women, its causes and consequences; UN General Assembly; UN Doc. A/67/227.

Maruzani, N. (2016). The Experiences of Visually Impaired Female Students in Selected Zimbabwe State Universities. Masters Dissertation. Masvingo. Great Zimbabwe University.

Maruzani, N. and Mapuranga, B. (2016). Gender and disability: a review of available literature. Research on Humanities and Social Sciences. 6(6). Pp. 74-86.

Matthews, G. F. (1983). Voices from the shadow: Women with disabilities speak out. Toronto, Ontario: The Women's Educational Press.

Mitra, S., Posarac, A., and Vick, B. (2013). Disability and poverty in developing countries: a multidimensional study. World Development, 41, 1-18.

Moyo, D.S. (2010). Ensuring sexual and reproductive health rights of women with disabilities: A study of policies, actions and commitments in Uganda and Zimbabwe. Masters Dissertation. Graduate Institute of International Development and Applied Economics. University of Reading.

Mtetwa, E. (2014). Disability policy and practice in Zimbabwe. In Mugumbate J., Dodo, O., Maushe, F., Ruparanganda, L., Zembere, M., Makwerere, D., Ngwerume, E. and Mtetwa, E. Development Policy and Practice: Making use of population census data in Zimbabwe. Harare. Institute of Development Administration.

Oliver, M. (1996) Understanding disability: From Theory to Practice. United Kingdom: Macmillan.

Palmer, M and Harley, D. (2012) Models and measurement in disability: an international review. http://heapol.oxfordjournals.org/content/27/5/357.full.pdf + html. [Accessed:29 November 2015].

Peta, C. (2015). Voices from the periphery: a narrative study of the experiences of sexuality of disabled women in Zimbabwe: PhD thesis. Cape Town. University of Cape Town.

Scheerenberger, R. C. (1987). A history of mental retardation: A quarter century of promise. Baltimore, MD: Paul H. Brookes.

Shaul, S., Dowling, P. J., and Laden, B. F. (1985). Like other women: Perspectives of mothers with physical disabilities. In M. J. Deegan \& N. A. Brooks (Eds.), Women and disability: The double handicap. New Brunswick, NJ: Transaction Books.

Simmons, H. G. (1982). From asylum to welfare. Toronto, Ontario, Canada: National Institute on Mental Retardation.

Waxman, B. F. (1989). The politics of sex and disability. Disability Studies Quarterly, 9 (3). Pp 1-5.

World Health Organization. (2001). International classification of functioning, disability and 
health.http://www.who.int/classifications/icf/en/ .[Accessed:1 $11^{\text {th }}$ December 2015].

World Health Organization (WHO) (2011).World Report on Disability, World Health Organization. Geneva, Switzerland.

Women With Disabilities, Human Rights Watch (HRW), Open Society Foundations, and the International Disability Alliance (IDA) (2011) Sterilization of Women and Girls with Disabilities: A Briefing Paper. Available at: Error! Hyperlink reference not valid..

Zinanga, E. (1996). Sexuality, identity and change. Southern Africa Feminist Review, 2(1), 36. 